EESTI NSV TEADUSTE AKADEEMIA TOIMETISED. XI KÖDE

FOOSIKALIS-MATEMAATILISTE JA TEHNILISTE TEADUSTE SEERIA, 1962, NR. I

ИЗВЕСТИЯ АКАДЕМИИ НАУК ЭСТОНСКОИ ССР. ТОМ ХI

СЕРИЯ ФИЗИКО-МАТЕМАТИЧЕСКИХ И ТЕХНИЧЕСКИХ НАУК. 1962, № 1

\title{
О НЕКОТОРЫХ ОБОБЩЕНИЯХ ТЕОРЕМЫ МЕРСЕРА ДЛЯ ДВОЙНЫХ ПОСЛЕДОВАТЕЛЬНОСТЕЙ
}

\section{т. СЫРмуС}

Настоящая статья является продолжением статьи [3], в которой мы указали, что два основных встречающихся вида теоремы Мерсера (теоремы I и II из [3]) не тождественны. В связи с этим интересно отметить, что результаты, получаемые при обобшении теоремы Мерсера на случай двойных последовательностей, различны в зависимости от того, которая из теорем I и II или ее обобщений берется в основу. Целью настоящей статьи является показать, в чем состоит различие этих результатов и доказать ряд обобщений теоремы Мерсера для двойных последовательностей.

Пусть $P^{\alpha, \beta}$ - факторизирующаяся матрища преобразования двойной последовательности в двойную последовательность и $E$ - матрица тождественного преобразования.

Мы будем называть преобразование последовательности, определенное матрицей вида $\lambda E+(1-\lambda) P^{\alpha, \beta}, \quad$ двучленным преобразованием Мерсера $-P^{\alpha, \beta}$. Аналогично назовем преобразование последовательности, определенное матрицей вида $\lambda_{1} \lambda_{2} E+\left(1-\lambda_{1}\right) \lambda_{2} P^{\alpha, 0}+\lambda_{1}\left(1-\lambda_{2}\right) P^{0, \beta}+\left(1-\lambda_{1}\right)\left(1-\lambda_{2}\right) P^{\alpha, \beta}$, четырехчленным преобразованием Мерсера $-P^{\alpha, \beta}$.

Для случая четырехчленного преобразования * Мерсера $-H^{1,1}$ имеются два доказательства теоремы Мерсера, принадлежащие одна Мерсеру [12], а другая Раманужану [14]. Они доказывают теоремы, отличающиеся друг от друга так же, как наши теоремы I и II из [3].

Теорема Мерсера для двучленного преобразования Мерсера - взвешенных средних Рисса, а следовательно, и для преобразования Мерсера - $H^{1,1}$ доказана в [ $\left.{ }^{2}\right]$.

Из $\left[2,{ }^{14}\right]$ и результатов настоящей статьн следует, что в основе теорем Мерсера для четырехчленных преобразований $M-P^{\alpha, \beta}$ лежит теорема II. из [3] или ее обобщения, а в основе теорем Мерсера для двучленных преобразований $M-P^{\alpha, \beta}$ лежит теорема I из $\left[{ }^{3}\right]$ или ее обобщения.

В первом параграфе нашей статьи мы введем символику, приведем необходимые нам теоремы и докажем ряд лемм. Во втором параграфе докажем теорему Мерсера для двучленного преобразования Мерсера - $\left(H, \mu_{k l}\right)$, ограничиваясь факторизирующимися преобразованиями Хаусдорфа. Здесь же дадим следствия доказанной теоремы. В третьем параграфе докажем теорему Мерсера для четырехчленных преобразований Мерсера $-C^{\alpha, \beta}$ и Мерсера $-H^{\alpha, \beta}$.

- Определения методов Гелдера $H^{\alpha, \beta}$ даны в $\left.{ }^{[}{ }^{3}\right]$ и [9], Чезаро $C^{\alpha, \beta}-$ в $\left[{ }^{8}\right]$ и $\left[{ }^{13}\right]$, Хаусдорфа $\left(H, \mu_{k l}\right)-$ в [7] и $\left[{ }^{14}\right]$, взвешенных средних Рисса $\left(R, p_{k l}\right)-$ B [11]. 
1. Следуя Адамсу [5], обозначим факторпзирующиеся преобразования Хаусдорфа символом $\left(H, \mu_{k}\right) \odot\left(H, v_{l}\right)=\left(H, \mu_{k l}\right)$, где $\mu_{k l}=\mu_{k} v_{l}$. Матричные методы будем обозначать через $A=\left(a_{m n k l}\right)$ и т. д., а обратные им, соответственно, через $A-1=\left(a_{m n k l}^{\prime}\right)$ и т. д. Для конечных разностей воспользуемся общепринятыми символами. Следуя Гамильтону $\left[{ }^{10}\right]$, пусть $b-$ класс ограниченных двойных последовательностей, $c$ - класс сходящихся двойных последовательностей, $b c-$ класс сходящихся-и ограниченных дзойных последовательностей.

В настоящей работе нам нужны сяедующие теоремы.

Т еорем а 1.1. (Польняковский [']).

В случае методов ${ }^{*} C^{\alpha}$ и $H^{\alpha}, c \alpha=2,3, \ldots$, суцествуют постоянные, соответственно, $\lambda_{\alpha}^{\prime}$ и $\lambda_{\alpha}^{\prime \prime}$ такие, что из условия

$$
\lim _{n}\left(\lambda s_{n}+(1-\lambda) t_{n}\right)=h,
$$

где $t_{n}$ ознанает, соответственно, $C_{n}^{\alpha}$ или $H_{n}^{\alpha}$, следует $\lim _{n} s_{n}=h$ тогда и только тогда. когда $\lambda>\lambda_{\alpha}^{\prime}$ или $\lambda>\lambda_{\alpha}^{\prime \prime}$. Обе последовательности, $\left\{\lambda_{\alpha}^{\prime}\right\}$ и $\left\{\lambda_{\alpha}^{\prime \prime}\right\}$, являются возрастаюцими при $\alpha \geqslant 2$, неотрицательными и сходяцимися $\kappa$ значению $\frac{1}{2}$.

Справедлиаы слео̆ующие асимптотические представления:

$$
\begin{gathered}
\lambda_{\alpha}^{\prime} \sim \frac{1}{2}-\frac{1}{48} \frac{\pi^{4}}{\ln ^{2} \alpha}, \\
\lambda_{\alpha}^{\prime \prime}=\frac{\cos ^{\alpha} \frac{\pi}{a}}{1+\cos ^{\alpha} \frac{\pi}{\alpha}} \sim \frac{1}{2}-\frac{\pi^{2}}{8 \alpha} .
\end{gathered}
$$

Tеорем а 1.2. (Адамс ['], теорема 1L).

Если $A^{\prime}$ и $A^{\prime \prime}$ - регулярные методы простых последовательностей, то метод $A=A^{\prime} \odot A^{\prime \prime}$ регулярен в этом классе двойных последовательностей, А-преобразовани: котирых ограничены.

Т е о рем а 1.3. (Адамс [6], теорема 7).

Если $A^{\prime}$ и $A^{\prime \prime}$ обратимые методы простых последовательностей, то метод $A=A^{\prime} \odot A^{\prime \prime}$ имеет обратный метод вида $A^{-1}=\left(A^{\prime}\right)^{-1} \odot\left(A^{\prime \prime}\right)^{-1}$.

Докажем теперь следующие леммы.

Л ем а 1.1. Если $\left(H, \mu_{m n}\right)$-преобразование последовательности $\left\{s_{m n}\right\}$ ограничено, а метод $\left(H, \mu_{m n}\right)^{-1}$ сохраняет ограниченность, то и сама последовательность $\left\{s_{\text {mи }}\right\}$ ограничена

Доказ ательство. По условию метод $\left(H, \frac{1}{\mu_{m n}}\right)$ сохраняет ограниченность. следовательно, существуют такие постоянные $L$ и $M$, что

$$
\sum_{k, l=0}^{m, n}\left(\begin{array}{l}
m \\
k
\end{array}\right)\left(\begin{array}{l}
n \\
l
\end{array}\right)\left|\Delta^{m-k, n-l} \frac{1}{\mu_{k l}}\right| \leqslant L \quad(m, n=0,1,2, \ldots)
$$

и, кроме того,

* Определения методов Чезаро $C^{\alpha}$ и Гелдера $H^{\alpha}$ даны в [ $\left.{ }^{4}\right] ;$ символ $\lim _{n} x_{n}$ означает $\lim x_{n}$ 


$$
\left|t_{m n}\right|=\left|\sum_{k, l=0}^{m, n}\left(\begin{array}{l}
m \\
k
\end{array}\right)\left(\begin{array}{l}
n \\
l
\end{array}\right) \Delta^{m-k, n-l} \mu_{k l} s_{k l}\right| \leqslant M \quad(m, n=0,1,2, \ldots)
$$

а поэтому

$$
\left|s_{m n}\right| \leqslant \sum_{k, l=0}^{m, n}\left(\begin{array}{l}
m \\
k
\end{array}\right)\left(\begin{array}{l}
n \\
l
\end{array}\right)\left|\Delta m-k, n-l \frac{1}{\mu_{k l}}\right|\left|t_{k l}\right| \leqslant M L \quad(m, n=0,1,2, \ldots),
$$

что и требовалось доказать.

Л ем м 1.2. Если $\lambda>\lambda_{\alpha}^{\prime \prime}$, где $\lambda_{\alpha}^{\prime \prime}=\frac{\cos ^{\alpha} \frac{\pi}{a}}{1+\cos ^{\alpha} \frac{\pi}{\alpha}} u \alpha=2,3, \ldots$,

то последовательность Хаусдорфа $\mu_{k}=\frac{1}{\lambda+(1-\lambda) \frac{1}{(k+1)^{\alpha}}}$ регулярна.

Доказательство. Из теоремы 1.1 следует, что метод $\lambda E+(1-\lambda) H^{\alpha}$ при $\lambda>\lambda_{\alpha}$ " равносилен тождественному. По теоремам 209 и 208 из [4] это означает, что при сделанных относительно $\lambda$ и $\lambda_{\alpha}^{\prime \prime}$ предположениях последовательность $\mu_{k}=\frac{1}{\lambda+(1-\lambda) \frac{1}{(k+1)^{\alpha}}}-$ регулярная последовательность Хаусдорфа, что и требовалось доказать.

Нижеследующие две леммы доказываются аналогично. При этом в доказательстве леммы 1.4 используем вместо теоремы 1.1 теорему 3.1 из [3].

Л м м а 1.3. Если $\lambda>\lambda_{\alpha}^{\prime}$, где $\lambda_{\alpha}^{\prime}$ определено как в теореме $1.1 u \alpha=2,3, \ldots$, то

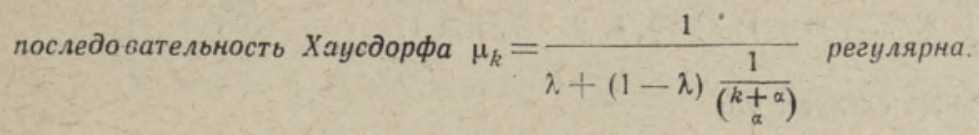

Л емм а 1.4. Если $\lambda>0$ и $0<\alpha<1$, то последовательность Хаусдорфа $\mu_{k}=\frac{1}{\lambda+(1-\lambda) \frac{1}{(k+1)^{\alpha}}}$ регулярна.

2. В этом ліараграфе докажем для методов Хаусдорфа вида $\left(H, \mu_{k}\right) \odot\left(H, v_{l}\right)=$ $=\left(H, \mu_{p l}\right)$ следующее обобщение теоремы Мерсера.

Т еорем а 2.1. Если $\lambda>0, h \neq \infty$, последовательности Хаусдорфа $\left\{\mu_{k}\right\} u\left\{v_{b}\right\}$ абсолютно монотонны $и$ удовлетворяют условиям $\lim _{m} \Delta^{m} \mu_{0}=\lim _{n} \Delta^{n} v_{0}=0 u \mu_{0} v_{0}=1$, то из равенства *

$$
b-\lim _{m, n}\left(\lambda s_{m n}+(1-\lambda) \sum_{k, l=0}^{m-1, n-1}\left(m_{k}-1\right)(n-1) \Delta^{m-1-k, n-1-l} \mu_{k} v_{l} s_{k l}\right)=h
$$

вытекает

$$
b-\lim _{m, n} s_{m n}=h \text {. }
$$

* Под снмволом $b-\lim _{m, n} S_{m n}=S$ мы подразумеваем $\lim _{m, n} S_{m n}=S$ при $S_{m n}=O(1)$. 
Доказ ательство. Пусть

$$
t_{m n}=\lambda s_{m n}+(1-\lambda) H_{m-1, n-1}
$$

или

$$
t_{m n}=\lambda \sum_{k, l=0}^{m, n} h_{m n k l}^{\prime} H_{k l}+(1-\lambda) H_{m-1, n-1},
$$

где

$$
h_{m n k l}=\left(\begin{array}{c}
m \\
k
\end{array}\right)\left(\begin{array}{l}
n \\
l
\end{array}\right) \Delta m-k, n-l \mu_{k l} \text { " } H_{m n}=\sum_{k, l=0}^{m, n} h_{m n k l} s_{k l}
$$

Вви,уу леммы 2.1 из $\left[{ }^{2}\right]$ результат обращения равенства $(2.2)$ имеет вид

$$
H_{m n}=\frac{\sum_{k, l=0}^{m, n} \gamma_{m n k l} t_{k l}}{\sum_{k, l=0}^{m, n} \gamma_{m n k l}}
$$

где $\gamma_{m n m n}-$ произвольная величина, а остальные $\gamma_{m n k l}$ определяются снстемой

$$
\begin{aligned}
& \sum_{l=s}^{n} l_{m n m i}\left(\begin{array}{l}
l \\
s
\end{array}\right) \Delta^{l-s} \frac{1}{v_{s}}=0 \quad(s=0,1,2, \ldots, n-1), \\
& \sum_{k=r}^{m} \gamma_{m i n k n}\left(\begin{array}{l}
k \\
r
\end{array}\right) \Delta^{k-r} \frac{1}{\mu_{r}}=0 \quad(r=0,1,2, \ldots, m-1), \\
& \sum_{k, l=r, s}^{m, n} \gamma_{m n k l}\left(\begin{array}{l}
k \\
r
\end{array}\right)\left(\begin{array}{l}
l \\
s
\end{array}\right) \Delta^{k-r, l-s} \frac{1}{\mu_{r} v_{s}}+\frac{1-\lambda}{\lambda} \gamma_{m n, r+1, s+1}=0 \\
& (r=0,1,2, \ldots, m-1 ; s=0,1,2, \ldots, n-1) .
\end{aligned}
$$

Из леммы $2.1\left[{ }^{2}\right]$ также следует, что

$$
\sum_{k, l=0}^{m, n} \gamma_{m n k l}=\frac{\lambda \gamma_{0}}{\mu_{m} v_{n}}
$$

в котором $\gamma_{0}=\gamma_{m n m n}$.

Обознатив $c=\frac{1-\lambda}{\lambda}$, прнведем полученную систему к виду

$\sum_{l=s}^{n-1} \gamma_{m n m l}\left(\begin{array}{l}l \\ s\end{array}\right) \Delta^{i-s} \frac{1}{v_{s}}=-\left(\begin{array}{l}n \\ s\end{array}\right) \Delta^{n-s} \frac{1}{v_{s}} \gamma_{0} \quad(s=0,1,2, \ldots, n-1)$,
$\sum_{k=r}^{m-1} \gamma_{m n k n}\left(\begin{array}{l}k \\ r\end{array}\right) \Delta^{k-r} \frac{1}{\mu_{r}}=-\left(\begin{array}{c}m \\ r\end{array}\right) \Delta^{m-r} \frac{1}{\mu_{r}} \gamma_{0} \quad(r=0,1,2, \ldots, m-1)$.

$\sum_{k, l=r, s}^{m-1, n-1} \gamma_{m r_{i} k l} \mu_{r} v_{s}\left(\begin{array}{l}l \\ s\end{array}\right)\left(\begin{array}{l}k \\ r\end{array}\right) \Delta^{k-r, l-s} \frac{1}{\mu_{r} v_{s}}=\mu_{r} v_{s} \gamma_{0}\left(\begin{array}{l}m \\ r\end{array}\right)\left(\begin{array}{l}n \\ s\end{array}\right) \Delta^{m-r, n-s} \frac{1}{\mu_{r} v_{s}}-$ $-c \mu_{r} v_{s} \gamma_{m n, r+1, s+1}(r=0,1,2, \ldots, m-1 ; s=0,1,2, \ldots, n-1)$. 
Выделяя из (2.6) систему

$$
\sum_{l=s}^{n-1} \gamma_{m n m l}\left(\begin{array}{l}
l \\
s
\end{array}\right) \Delta^{l-s} \frac{1}{v_{s}}=-\left(\begin{array}{l}
n \\
s
\end{array}\right) \Delta^{n-s} \frac{1}{v_{s}} \gamma_{0}(s=0,1,2, \ldots, n-1)
$$

и сравнивая ее с системой (2.9) из [3], мы виднм, что этн две системы тождественны. Это же справедливо для системы

$$
\sum_{k=r}^{m-1} \gamma_{m n k n}\left(\begin{array}{l}
k \\
r
\end{array}\right) \Delta^{k-r} \frac{1}{\mu_{r}}=-\left(\begin{array}{l}
m \\
r
\end{array}\right) \Delta^{m-r} \frac{1}{\mu_{r}} \gamma_{0} \quad(r=0,1,2, \ldots, m-1) .
$$

Следовательно, решения этих двух систем совпадают с решением системы (2.9) из [3], т. е. имеем

$$
\left\{\begin{array}{l}
\gamma_{m n m l}=\gamma_{0} \frac{1}{v_{n}}\left(\begin{array}{c}
n \\
l
\end{array}\right) \Delta^{n-l} v_{l} \quad(l=0,1,2, \ldots, n-1) \\
\gamma_{m n k n}=\gamma_{0} \frac{1}{\mu_{m}}\left(\begin{array}{c}
m \\
k
\end{array}\right) \Delta m-k \mu_{k} \quad(k=0,1,2, \ldots, m-1) .
\end{array}\right.
$$

Для решения оставшейся части системы (2.6) представим искомое $\gamma_{m n k l}$ суммой двух слагаемых, т. е.

$$
\gamma_{m n k l}=X_{m n k l}+Y_{m n k l}
$$

требуя, чтобы слагаемые удовлетворяли системам

$$
\left\{\begin{array}{l}
\sum_{k, l=r, s}^{m-1, n-1} X_{m n k l}\left(\begin{array}{l}
k \\
r
\end{array}\right)\left(\begin{array}{l}
l \\
s
\end{array}\right) \Delta k-r, l-s \frac{1}{\mu_{r} v_{s}}=\gamma_{0}\left(\begin{array}{c}
m \\
r
\end{array}\right)\left(\begin{array}{l}
n \\
s
\end{array}\right) \Delta m-r, n-s \frac{1}{\mu_{r} v_{s}} \\
(r=0,1,2, \ldots, m-1 ; s=0,1,2, \ldots, n-1)
\end{array}\right.
$$

и

$$
\left\{\begin{array}{l}
\sum_{k, l=r, s}^{m-1, n-1} Y_{m n k l} \mu_{r} v_{s}\left(\begin{array}{l}
k \\
r
\end{array}\right)\left(\begin{array}{l}
l \\
s
\end{array}\right) \Delta k-r, l-s \frac{1}{\mu_{r} v_{s}}=-c \mu_{r} v_{s} \gamma_{m n, r+1, s+1} \\
(r=0,1,2, \ldots, m-1 ; s=0,1,2, \ldots, n-1)
\end{array}\right.
$$

Решение систем (2.9) имеет вид

$$
\begin{gathered}
X_{m n k l}=\gamma_{0} \frac{1}{\mu_{m} v_{n}}\left(\begin{array}{c}
m \\
k
\end{array}\right)\left(\begin{array}{l}
n \\
l
\end{array}\right) \Delta m-k, n-l \mu_{k} v_{l} \\
(k=0,1,2, \ldots, m-1 ; l=0,1,2, \ldots, n-1) .
\end{gathered}
$$

Находим это решение аналогично тому, как было найдено решение системы (2.9) из [3].

Путем элементарных, но объемистых вычислений можно показать, применяя формулу (1.3) из [3], что решение системы (2.10) сводится к решению следуюшего ряда систем:

a) первая группа систем получается при $r=0,1,2, \ldots, m-1$ из системы

$$
\begin{gathered}
\sum_{l=s}^{n-(m-r)} Y_{m n i r l} v_{s}\left(\begin{array}{l}
l \\
s
\end{array}\right) \Delta l-s \frac{1}{v_{s}}=-\sum_{l=n-(m-r)+1}^{n-1} Y_{m n r l} v_{s}\left(\begin{array}{l}
l \\
s
\end{array}\right) \Delta l-s \frac{1}{v_{s}}- \\
-c v_{s} \sum_{k=r}^{m-1}\left(\begin{array}{l}
k \\
r
\end{array}\right) \Delta k-r \mu_{r} \gamma_{m n, k+1, s+1} \quad(s=0,1,2, \ldots, n-(m-r))
\end{gathered}
$$



снстемы

б) вторая группа систем получается при $s=n-m, n-(m-1), \ldots, n-1$ из

$$
\begin{aligned}
& \sum_{k=r}^{m-(n-s)} Y_{m r_{k} k s} \mu_{r}\left(\begin{array}{l}
k \\
r
\end{array}\right) \Delta^{k-r} \frac{1}{\mu_{r}}=-\sum_{k=m-(n-s)+1}^{m-1} Y_{m n k, s} \mu_{r}\left(\begin{array}{l}
k \\
r
\end{array}\right) \Delta^{k-r} \frac{1}{\mu_{r}}- \\
& -c \mu_{r} \sum_{l=s}^{n-1}\left(\begin{array}{l}
l \\
s
\end{array}\right) \Delta l-s v_{s} \gamma_{m n, r+1, l+1} \quad(r=0,1,2, \ldots, m-(n-s)) .
\end{aligned}
$$

При получении этих систем сделано предположение $n \geqslant m$, что, конечно, не ограничивает общности.

Решая полученные системы аналогично тому, как была решена система (2.10) нз [3], можно путем элементарных, но объемистых вычислений доказать, что система (2.10) имеет следующее решение:

a) при $k=0,1,2, \ldots, m-1$

$$
\begin{gathered}
Y_{m n k l}=-c \gamma_{0} \sum_{\sigma_{0}==k, l}^{m-1, n-1} \frac{1}{\mu_{m}}\left(\begin{array}{c}
m+1 \\
\sigma+1
\end{array}\right) \Delta^{m-(\sigma+1)} \mu_{\sigma+1}\left(\begin{array}{l}
\sigma \\
k
\end{array}\right) \Delta^{\sigma-k} \mu_{k} . \\
\cdot \frac{1}{v_{n}}\left(\begin{array}{c}
n \\
\tau+1
\end{array}\right) \Delta^{n-(\tau+1)} v_{\tau+1}\left(\begin{array}{l}
i \\
i
\end{array}\right) \Delta^{\tau-l} v_{l}+
\end{gathered}
$$

$$
\begin{aligned}
& +c^{2} \gamma_{0} \sum_{\substack{\sigma_{1}=k \\
\tau_{1}=l}}^{m-2, n-2} \sum_{\substack{\sigma=\sigma_{1}+1 \\
\tau=\tau_{1}+1}}^{m-1, n-1} \frac{1}{\mu_{m}}\left(\begin{array}{c}
m+1 \\
\sigma+1
\end{array}\right) \Delta^{m-(\sigma+1)} \mu_{\sigma+1}\left(\sigma_{1}+1\right) \Delta^{\sigma-\left(\sigma_{1}+1\right)} \mu_{\sigma_{1}+1}\left(\begin{array}{c}
\sigma_{1} \\
k
\end{array}\right) \Delta^{\sigma_{1}-k} \mu_{k} . \\
& \cdot \frac{1}{v_{n}}\left(\begin{array}{c}
n \\
\tau+1
\end{array}\right) \Delta^{n-(\tau+1)} v_{\tau+1}\left(\tau_{\tau_{1}+1}+\Delta^{\tau-\left(\tau_{1}+1\right)} v_{\tau_{1}+1}\left(\begin{array}{c}
\tau_{1} \\
l
\end{array}\right) \Delta^{\tau_{1}-l} v_{l}\right. \\
& -c^{3} \gamma_{0} \sum_{\substack{\sigma_{2}=k \\
=2=1}}^{m-3, n-3} \sum_{\substack{\sigma_{1}=\sigma_{2}+1 \\
\tau_{1}=\tau_{2}+1}}^{m-2, n-2} \sum_{\substack{\sigma=\sigma_{1}+1 \\
\tau=\tau_{1}+1}}^{m-1, n-1} \frac{1}{\mu_{m}}\left(\begin{array}{c}
m+1 \\
\sigma+1
\end{array}\right) \Delta^{m-(\sigma+1)} \mu_{\sigma+1}\left(\begin{array}{c}
\sigma \\
\sigma_{1}+1
\end{array}\right) . \\
& \cdot \Delta^{\sigma-\left(\sigma_{1}+1\right)} \mu_{\sigma_{1}+1}\left(\sigma_{2}+1\right) \Delta^{\sigma_{1}-\left(\sigma_{2}+1\right)} \mu_{\sigma_{2}+1} \text {. } \\
& \cdot\left(\begin{array}{c}
\sigma_{n} \\
k
\end{array}\right) \Delta^{\sigma_{2}-k} \mu_{k} \cdot \frac{1}{v_{n}}\left(\begin{array}{c}
n \\
\tau+1
\end{array}\right) \Delta^{n-(\tau+1)} v_{\tau+1}\left(\left(_{\tau_{1}}+1\right) \Delta^{\tau-\left(\tau_{1}+1\right)} v_{\tau_{1}+1}\left(\tau_{\tau_{2}+1}^{\tau_{1}+1}\right) \cdot\right. \\
& \cdot \Delta^{\tau_{1}-\left(\tau_{2}+1\right)} v_{\tau_{2}+1}\left(\bar{i}_{l}^{2}\right) \Delta^{\tau_{2}-l} v_{l}+\ldots \\
& \ldots+(-1)^{m-k} c^{m-k} \gamma_{0} \mu_{k} \mu_{k+1} \ldots \mu_{m-1} \sum_{\tau_{m-(k+1)}=l-(m-k)}^{n-\tau_{m-(k+2)}=\sum_{m-(k+1)}+1} \cdots \\
& \ldots \sum_{\tau=\tau+1}^{n-1} \frac{1}{v_{n}}\left(\begin{array}{c}
n+1 \\
\tau+1
\end{array}\right) \Delta^{n-(\tau+1)} v_{\tau+1}\left(\tau_{\tau_{1}+1}^{\tau}\right) \Delta^{\tau-\left(\tau_{1}+1\right)} v_{\tau_{1}+1} \ldots \\
& \ldots\left(\begin{array}{c}
\tau_{m-(k+2)} \\
\tau_{m-(k+1)}+1
\end{array}\right) \Delta^{\tau_{m-(k+2)-}\left(\tau_{m-(k+1)}+1\right)} v_{\tau_{m-(k+1)}}+1\left(\tau_{m-(k+1)}\right) \Delta^{\tau_{m-(k+1)}-l_{v}} \\
& (l=0,1,2, \ldots, n-(m-k)) ;
\end{aligned}
$$


б) ири $l=0,1,2, \ldots, n-1$

$$
\begin{aligned}
& Y_{m k k l}=-c \gamma_{0} \sum_{\sigma, \tau=k, l}^{m-1, n-1} \frac{1}{\mu_{m}}\left(\begin{array}{c}
m+1 \\
\sigma+1
\end{array}\right) \Delta^{m-(\sigma+1)} \mu_{\sigma+1}\left(\begin{array}{l}
\sigma \\
k
\end{array}\right) \Delta^{\sigma-k} \mu_{k} . \\
& \cdot \frac{1}{v_{n}}(\tau+1) \Delta^{n-(\tau+1)} v_{\tau+1}(\bar{i}) \Delta^{\tau-l} v_{t}+ \\
& +c^{2} \gamma_{0} \sum_{\substack{\sigma_{1}=k \\
\tau_{1}=l}}^{m-2, n-2} \sum_{\substack{\sigma=\sigma_{1}+1 \\
\tau=\tau_{1}+1}}^{m-1, n-1} \frac{1}{\mu_{m}}\left(\begin{array}{c}
m+1 \\
\sigma+1
\end{array}\right) \Delta^{m-(\sigma+1)} \mu_{\sigma+1}\left(\sigma_{\sigma_{1}+1}^{\sigma}\right) \Delta^{\sigma-\left(\sigma_{1}+1\right)} \mu_{\sigma_{1}+1}\left(\begin{array}{c}
\sigma_{1} \\
k
\end{array}\right) \Delta^{\sigma_{1}-k} \mu_{k} \cdot \\
& \cdot \frac{1}{v_{n}}\left(\begin{array}{c}
n \\
\tau+1
\end{array}\right) \Delta^{n-(\tau+1)} v_{\tau+1}\left(\tau_{\tau_{1}+1}^{\tau}\right) \Delta^{\tau-\left(\tau_{1}+1\right)} v_{\tau_{1}+1}\left(\tau_{l}^{\tau}\right) \Delta^{\tau_{1}-l} v_{l} \\
& =c^{3} \gamma_{0} \sum_{\substack{\sigma_{g}=k \\
\tau_{2}=l}}^{m-3, n-3} \sum_{\substack{\sigma_{1}=\sigma_{2}+1 \\
\tau_{1}=\tau_{2}+1}}^{m-2, n-2} \sum_{\substack{\sigma=\sigma_{1}+1 \\
\tau=\tau_{1}+1}}^{m-1, n-1} \frac{1}{\mu_{m}}\left(\begin{array}{c}
m+1 \\
\sigma+1
\end{array}\right) \Delta^{m-(\sigma+1)} \mu_{\sigma+1}\left(\begin{array}{c}
\sigma \\
\sigma_{1}+1
\end{array}\right) \Delta^{\sigma-\left(\sigma_{1}+1\right)} \mu_{\sigma_{1}+1} \text {. } \\
& \cdot\left(\begin{array}{c}
\sigma_{2}+1 \\
\sigma_{2}+1
\end{array}\right) \Delta^{\sigma_{1}-\left(\sigma_{2}+1\right)} \mu_{\sigma_{2}+1}\left(\begin{array}{c}
\sigma_{2} \\
k
\end{array}\right) \Delta^{\sigma_{2}-k} \mu_{k} \frac{1}{v_{n}}\left(\begin{array}{c}
n \\
\tau+1
\end{array}\right) \Delta^{n-(\tau+1)} \nu_{\tau+1} . \\
& \cdot\left(\tau_{\tau_{1}+1}\right) \Delta^{\tau-\left(\tau_{1}+1\right)} v_{\tau_{1}+1}\left(\tau_{\tau_{2}+1}^{\tau_{1}}\right) \Delta^{\tau_{l}-\left(\tau_{2}+1\right)} v_{\tau_{2}+1}\left(\tau_{l}^{\tau_{2}}\right) \Delta^{\tau-l} v_{l}+\ldots \\
& \cdots+(-1)^{n-l} c^{n-l} \gamma_{0} v_{l} \nu_{l+1} \ldots v_{n-1} \sum^{m-(n-t)} \sum^{m-(n-l)+1} \\
& \sigma_{n-(l+1)}=k \quad \sigma_{n-(l+2)}=\sigma_{n-(l+1)}+1 \\
& \ldots \sum_{\sigma=\sigma_{1}+1}^{m-1} \frac{1}{\mu_{m}}\left(\begin{array}{c}
m \\
\sigma+1
\end{array}\right) \Delta^{m-(\sigma+1)} \mu_{\sigma+1} \text {. } \\
& \cdot\left(\sigma_{1}+1\right) \Delta^{\sigma-\left(\sigma_{1}+1\right)} \mu_{\sigma_{1}+1} \cdots\left(\begin{array}{c}
\sigma_{n-(l+2)} \\
\sigma_{n-(l+1)+1}
\end{array}\right) \Delta^{\sigma_{n-(l+2)}}-\left(\sigma_{n-(l+1)}+1\right) \mu_{\sigma_{n-(l+1)}+1} \\
& \left(\underset{k}{\sigma_{n-(l+1)}}\right) \Delta^{\sigma_{n-(l+1)}}{ }_{\mu_{k}}^{-k}(k=0,1,2, \ldots, m-(n-l))
\end{aligned}
$$




$$
\begin{array}{rr}
b_{m n k, n-1}^{\prime}=-c v_{n-1}\left({ }_{k}^{m-1}\right) \Delta m-1-k \mu_{k} & (k=0,1,2, \ldots, m-2) \\
b_{m n, m-1, l}^{\prime}=-c \mu_{m-1}\left({ }^{n-1}\right) \Delta^{n-1}-v_{l} & (l=0,1,2, \ldots, n-2) \\
b_{m n k l}^{\prime}=-\frac{c}{\gamma_{0}} \mu_{m-1} v_{n-1} \gamma_{m-1, n-1, k l}(l=0,1,2, \ldots, n-(m-k) \\
k=0,1,2, \ldots, m-2) \\
b_{m n k l}^{\prime}=-\frac{c}{\gamma_{0}} \mu_{m-1} v_{n-1} \gamma_{m-1, n-1, k l} \begin{array}{r}
(k=0,1,2, \ldots, m-(n-l) \\
l=0,1,2, \ldots, n-2)
\end{array}
\end{array}
$$

где $\gamma_{m-1, n-1, k l}$ определены формулами (2.7), (2.11), (2.12) и (2.13).

Мы видим, что 1) $b_{m n k l}^{\prime}$ - полиномы относительно параметра $c$ и 2) перед четными степенями параметра $c$ стонт знак + , перед нечетными знак -

Зависимость элементов $b_{m n k l}^{\prime}$ и величин $\gamma_{m n k l}$ от параметра $c$ обозначим через $b_{m n k l}^{\prime}=b_{m n k l}^{\prime}(c) \quad$ и $\quad \gamma_{m n k l}=\gamma_{m n k l}(c)$.

Покажем теперь, что последовательность $\left\{t_{m n}\right\}$ из (2.1) может сходиться только тогда, когда сходится $\left\{s_{m n}\right\}$. Для этого убедимся, что условие (7) теоремы 2.2 из $\left[{ }^{2}\right]$ не. выполнлется, т. е. что

$$
\sup _{m, n} \sum_{k, l=0}^{m, n}\left|b_{m n k l}^{\prime}\right| \neq \infty
$$

Рассмотрим здесь два случая.

1) Пусть $c<0$, т. е. $\lambda>1$. По формулам (2.15) и замечаниям, сделанным относительно этих формул, ясно, что в этом случае $b_{m n k l}^{\prime}>0$. Из (2.14) теперь следует, что

$$
\begin{gathered}
\sum_{m, n=0}^{p, q}\left|b_{p q m n}^{\prime}\right|=\sum_{m, n=0}^{p, q} b_{p q m n}^{\prime}=\sum_{m, n=0}^{p, q} \sum_{k, l=m, n}^{p, q} h_{p q k l}^{\prime} \frac{\gamma_{k l m n}}{\sum_{m, n=0}^{k, l} \gamma_{k l m n}}= \\
=\sum_{k, l=0}^{p, q} h_{p q k l}^{\prime} \sum_{m, n=0}^{k, l} \frac{\sum_{m, n=0}^{\gamma_{k l m n}}}{\sum_{m=1}^{k, l} \gamma_{k l m n}}=\sum_{k, l=0}^{p, q} h_{p q k l}^{\prime}=1,
\end{gathered}
$$

ибо $\sum_{k, l=0}^{p, q} h_{p q k l}=1$ по условням нашей теоремы. Но это н приводит к (2.16).

2) Пусть $c>0$, т. е. $0<\lambda<1$. В этом случае

$$
\left|b_{m n k l}^{\prime}(c)\right| \leqslant b_{m n k l}^{\prime}(-c) \text {, }
$$

что вытекзет из $(2.15)$, и мы получаем

$$
\begin{gathered}
\sum_{m, n=0}^{p, q}\left|b_{p q n i n}^{\prime}(c)\right| \leqslant \sum_{m, n=0}^{p, q} b_{p q m n}^{\prime}(-c)=\sum_{m, n=0}^{p, q} \sum_{k, l=m, n}^{p, q} h_{p q k l}^{\prime} \frac{\gamma_{k l m n}(-c)}{\sum_{m, n=0}^{k, l} \gamma_{k l m n}(-c)}= \\
=\sum_{k, l=0}^{p, q} h_{p q k l}^{\prime} \sum_{m, n=0}^{k, l} \frac{\gamma_{k l m n}(-c)}{\sum_{m, n=0}^{k, l} \gamma_{k l m n}(-c)}=1 .
\end{gathered}
$$


Это также прнводит к (2.16). Следовательно, последовательность $\left\{t_{m n}\right\} \in b c$ только тогда, когда $\left\{s_{m n}\right\} \in c$. То, что $\left\{s_{m n}\right\} \in b$, вытекает из (2.16) и леммы 1.1. Этим теорема доказана.

Так как последовательности Хаусдорфа для методов $H^{\gamma, \beta}$ и $C^{\alpha, \beta}$ при $\alpha, \beta>0$ удовлетворяют условиям нашей теоремы* 2.1, то понятно, что имеют место две следующие теоремы.

Теорем а 2.2. Eсли $\lambda>0, h \neq \infty, \alpha, \beta>0 u$

$$
b-\lim _{m, n}\left(\lambda s_{m n}+(1-\lambda) C_{m-1, n-1}^{\alpha, \beta}\right)=h,
$$

ro

$$
b-\lim _{m, n} s_{m n}=h .
$$

Теорем а 2.3. Если $\lambda>0, h \neq \infty, \alpha, \beta>0 \quad u$

$$
b-\lim _{m, n}\left(\lambda s_{m n}+(1-\lambda) H_{m-1, n-1}^{\alpha, \beta}\right)=h
$$

To

$$
\text { b. } \lim _{m, n} s_{m n}=h
$$

Этим доказаны обобщения теоремы 1.1 из настоящей статьи и теоремы 2.1 из [3] для двойных последовательностей.

3. В этом параграфе мы покажем, что в основе теорем Мерсера для четырехчленных преобразований $M-H^{\alpha, \beta}$ и $M-C^{\chi, \beta}$ лежат теоремы 3.1 из [3] и 1.1 .

Теорем а 3.1. Если $\alpha, \beta=2,3, \ldots, h \neq \infty, \lambda_{1}>\lambda_{\alpha}, \lambda_{2}>\lambda_{\beta}$, где $\lambda_{\alpha}$ и $\lambda_{\beta}$ определены в теореме 1.1 u

$$
b-\lim _{m, n}\left(\lambda_{1} \lambda_{2} s_{m n}+\left(1-\lambda_{1}\right) \lambda_{2} C_{m n}^{\alpha, 0}+\left(1-\lambda_{2}\right) \lambda_{1} C_{m n}^{0, \beta}+\left(1-\lambda_{1}\right)\left(1-\lambda_{2}\right) C_{m n}^{\alpha, \beta}\right)=h,
$$

To

$$
b-\lim _{m, n} s_{m n}=h .
$$

Даказательство. Покажем, что преобразование $t_{m n}$ последовательности $\left\{s_{m n}\right\}$, где

$$
t_{m n}=\lambda_{1} \lambda_{2} s_{m n}+\left(1-\lambda_{1}\right) \lambda_{2} C_{m n}^{\alpha, 0}+\lambda_{1}\left(1-\lambda_{2}\right) C_{m n}^{0, \beta}+\left(1-\lambda_{1}\right)\left(1-\lambda_{2}\right) C_{m n}^{\alpha, \beta},
$$

является $\left(H, \mu_{m n}\right)$-преобразоьанием, которое определено нижеследующей матрицей Хаусдорфа:

где

$$
\left(H, \mu_{m n}\right)=\left(H, \mu_{m}\right) \odot\left(H, v_{n}\right),
$$

$$
\left\{\begin{array}{l}
\mu_{m}=\lambda_{1}+\frac{1-\lambda_{1}}{\left(\begin{array}{c}
m+\alpha \\
\alpha
\end{array}\right)} \\
\nu_{n}=\lambda_{2}+\frac{1-\lambda_{2}}{\left(\begin{array}{c}
n+\beta \\
\beta
\end{array}\right)} .
\end{array}\right.
$$

* См. конец $§ 2$ из [?]. 
Для этого вычислим произведение (3.2)

$$
\begin{gathered}
\left(H, \lambda_{1}+\frac{1-\lambda_{1}}{\left(\begin{array}{c}
m+\alpha \\
\alpha
\end{array}\right)} \odot\left(H, \lambda_{2}+\frac{1-\lambda_{2}}{\left(\begin{array}{c}
n+\beta \\
\beta
\end{array}\right)}\right)=\right. \\
=\left(( \begin{array} { c } 
{ m } \\
{ k }
\end{array} ) \Delta ^ { m - k } ( \lambda _ { 1 } + \frac { 1 - \lambda _ { 1 } } { ( \begin{array} { c } 
{ k + \alpha } \\
{ \alpha }
\end{array} ) } ) \odot \left(\left(\begin{array}{l}
n \\
l
\end{array}\right) \Delta^{n-l}\left(\lambda_{2}+\frac{1-\lambda_{2}}{\left(\begin{array}{l}
l+\beta \\
\beta
\end{array}\right)}\right)=\right.\right. \\
=\left(\lambda_{1} E^{1,0}+\left(1-\lambda_{1}\right) C^{\alpha, 0}\right) \odot\left(\lambda_{2} E^{0,1}+\left(1-\lambda_{2}\right) C^{0, \beta}\right)= \\
=\lambda_{1} \lambda_{2} E+\left(1-\lambda_{1}\right) \lambda_{2} C^{\alpha, 0}+\lambda_{1}\left(1-\lambda_{2}\right) C^{0, \beta}+\left(1-\lambda_{1}\right)\left(1-\lambda_{2}\right) C^{\alpha, \beta},
\end{gathered}
$$

где $E^{1,0}$ н $E^{0,1}$ - матрицы тождественного преобразования простых последовательностей.

Поэтому преобразование (3.1) перепишем в виде

$$
t_{m n}=\sum_{k, l=0}^{m, n}\left(\begin{array}{c}
m \\
k
\end{array}\right)\left(\begin{array}{l}
n \\
l
\end{array}\right) \Delta^{m-k, n-l}\left(\lambda_{1}+\frac{1-\lambda_{1}}{\left(\begin{array}{c}
k+\alpha \\
\alpha
\end{array}\right)}\right)\left(\lambda_{2}+\frac{1-\lambda_{2}}{\left(\begin{array}{c}
l+\beta \\
\beta
\end{array}\right)}\right) s_{k l}
$$

и отметим, что матрица, обратная матрице $(3.2)$, имеет вид $\left(H, \frac{1}{\mu_{m}}\right) \odot\left(H, \frac{1}{v_{n}}\right)$, как это следует из теоремы 1.3 .

Нужно показать, что метод (3.2) не может суммировать расходящиеся последовательности, т. е., что из ограниченной сходимости последовательности (3.3) вытекает сходимость $\left\{s_{m n}\right\}$. С этой целью произведем следующую оценку:

$$
\begin{aligned}
& \sum_{k, l=0}^{m, n}\left(\begin{array}{l}
m \\
k
\end{array}\right)\left(\begin{array}{l}
n \\
l
\end{array}\right)\left|\Delta m-k, n-l \frac{1}{\lambda_{1}+\frac{1-\lambda_{1}}{\left(\begin{array}{c}
k+\alpha \\
\alpha
\end{array}\right)}} \cdot \frac{1}{\lambda_{2}+\frac{1-\lambda_{2}}{\left(\begin{array}{c}
l+\beta \\
\beta
\end{array}\right)}}\right|=
\end{aligned}
$$

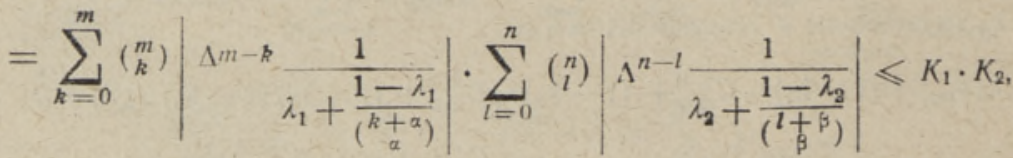

$$
\begin{aligned}
& (m, n=0,1,2 \ldots)
\end{aligned}
$$

что вытекает из леммы 1.3 .

Ввиду условия (3.4), заключаем по теоремам $(2.2)$ из $\left[^{2}\right]$ и 1.2 , что $\left\{s_{m n}\right\} \in c$. То, что $\left\{s_{m n}\right\} \in b$, вытекает из (3.4) н леммы 1.1. Равенство $b-\lim _{m, n} t_{m n}=b-\underset{m, n}{b} s_{m n}$ теперь очевндно.

\section{Теорема доказана.}

Аналогично можно доказать нижеследующие три теоремы, применяя при этом вместо леммы 1.3 , соответственно, леммы $1.2,1.4$ и одновременно 1.2 и 1.4 .

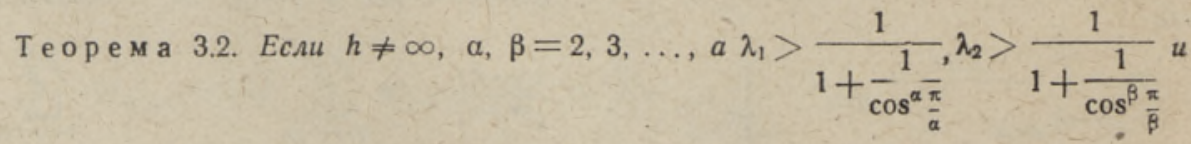

$$
b-\lim _{m, n}\left(\lambda_{1} \lambda_{2} s_{m n}+\left(1-\lambda_{1}\right) \lambda_{2} H_{m n}^{\alpha, 0}+\lambda_{1}\left(1-\lambda_{2}\right) H_{m n}^{0, \beta}+\left(1-\lambda_{1}\right)\left(1-\lambda_{2}\right) H_{m n}^{\alpha, \beta}\right)=h,
$$

$$
b-\lim _{m, n} s_{m n}=h .
$$


Теорем а 3.3. Если $\lambda_{1}>0, \lambda_{2}>0, h \neq \infty, 0<\alpha<1,0<\beta<1$ u

To

$$
b-\lim _{m, n}\left(\lambda_{1} \lambda_{2} s_{m n}+\left(1-\lambda_{1}\right) \lambda_{2} H_{m n}^{\alpha, 0}+\lambda_{1}\left(1-\lambda_{2}\right) H_{m n}^{0, \beta}+\left(1-\lambda_{1}\right)\left(1-\lambda_{2}\right) H_{m n}^{\alpha, \beta}\right)=h,
$$

$$
b-\lim _{m, n} s_{m n}=h
$$

Теорем а 3.4. Если $\lambda_{1}>0, \lambda_{2}>\frac{1}{1+\frac{1}{\cos ^{\beta} \frac{\pi}{\beta}}}, h \neq \infty, 0<\alpha<1, \beta=2,3, \ldots u$

TO

$$
b-\lim _{m, n}\left(\lambda_{1} \lambda_{2} s_{m n}+\left(1-\lambda_{1}\right) \lambda_{2} H_{m n}^{\alpha, 0}+\left(1-\lambda_{2}\right) \lambda_{1} H_{m n}^{0, \beta}+\left(1-\lambda_{1}\right)\left(1-\lambda_{2}\right) H_{m n}^{\alpha, \beta}\right)=h .
$$

$b-\lim _{m, n} s_{m n}=h$.

\section{Л ИТЕ Р А Т Р А}

1. 3. Польняковски й, О некоторых теоремах типа Мерсера. Бюл. Польской АН, 1956, отд. 3 , 4, № 5, 239-242.

2. Т. С ырмус, Об одном обобщении теоремы Мерсера для двойных последовательностей. Уч. зап. Тартуск. гос. ун-та, 102, 1961, 156-168.

3. Т. С ырм ус, О некоторых обобщениях теоремы Мерсера. Уч. зап. Тартуск. гос. ун-та, $102,1961,169-184$.

4. Г. Х а рд и, Расходящиеся ряды, М., ИЛ, 1949.

5. C. R. A d a m s, Transformations of double sequences with applications to Cesàro summability of double series. Bull Amer. Math. Soc., 37, No. 10, 1931, 741-748.

6. C. R. Ad a m s, On summability of double series. Trans. Amer. Math. Soc., 34, No. 2 , 1932, 215-230.

7. C. R. Ad a m s, Hausdorff transformations for double sequences. Bull. Amer. Math. Soc., 39, 1933, 303-312.

8. R. P. A g n ew, On summability of double sequences. Amer. J. Math., 54, 1932 , $648-656$.

9. T. J. B r o m w i c h, G. H. H a r d y, Some extensions to multiple series of Abel's theorem on the continuity of power series. Proc. London Math. Soc., 2, No. 2, 1904 , $161-189$.

10. H. J. Hamilto n, Transformations of multiple sequences. Duke Math. J., 2, No. 1, 1936, 29-60.

11. J. G. K u 11, Kahekordsete summeeruvate ridade korrutamine, Диссертация, Тартуск. гос. ун-т; см. также Уч. зап. Тартуск. гос. ун-та, $62,1958,3-59$.

12. J. Mercer, On the limit of real variants. Proc. London Math. Soc., (2), 1907, 5, 206-224.

13. Ch. N. Mo or e, Sur les facteurs de convergence dans les séries doubles et sur la série double de Fourier. C. r. Acad. sci. 155, 1912, 126-129.

14. M. S. R a m a n u ja n, On Hausdorff transformations for double sequences. Proc. Indian Acad. Sci. No. 3, 1955, 42. 


\section{MONINGATEST MERCERI TEOREEMI OLDISTUSTEST KAHEKORDSETE JADADE JAOKS}

\section{T. Sõrmus}

Töö olulisemaks tulemuseks on tuntud Merceri $\left[{ }^{12}\right]$ teoreemi järgmine üldistus:

Kui $\lambda>0, h \neq \infty$ ja Hausdorffi jadad $\left\{\mu_{k}\right\}$ ja $\left\{v_{l}\right\}$ on totaalselt monotoonsed ning rahuldavad tingimusi $\lim _{m} \Delta^{m} \mu_{0}=\lim _{n} \Delta^{n} v_{0}=0 j a v_{0} \mu_{0}=1$, siis võrdusest

$$
b-\lim _{m, n}\left(\lambda s_{m n}+(1-\lambda) \sum_{k, l=0}^{m-1, n-1}\left({ }_{k}^{m-1}\right)\left({ }^{n-1}\right) \Delta^{m-1-k, n-1-l} \mu_{k} v_{l} s_{k l}\right)=h
$$

järeldub, et $b-\lim _{m, n} s_{m n}=h$.

See teoreem on töös $\left[{ }^{3}\right]$ tõestatud teoreemi 2.1 analoog kahekordsetele jadadele ning temast saadakse erijuhuna Merceri teoreemi üldistused menetluste $C^{\alpha, \beta}$ ja $H^{\alpha, \beta} \quad(\alpha, \beta>0)$ jaoks (teoreemid 2.2 ja 2.3).

Ramanujan [14] on tõestanud Merceri teoreemi üldistuste kahekordsete jadade jaoks:

Kui $\lambda_{1}>0$ ja $\lambda_{2}>0$, siis võrdusest

$$
b-\lim _{m, n}\left(\lambda_{1} \lambda_{2} s_{m n}+\frac{\lambda_{1}\left(1-\lambda_{2}\right)}{n+1} \sum_{l=0}^{n} s_{m l}+\frac{\left(1-\lambda_{1}\right) \lambda_{2}}{m+1} \sum_{k=0}^{m} s_{l n}+\frac{\left(1-\lambda_{1}\right)\left(1-\lambda_{2}\right)}{(m+1)(n+1)} \sum_{k, l=0}^{m, n} s_{h l}\right)=h
$$

järeldub, et $b-\lim s_{m n}=h$.

$m, n$

Nagu töös näidatakse, osutub Ramanujani teoreem erijuhuks üldisematest teoreemidest, mis käsitlevad menetlusi $C^{\alpha, \beta}$ ja $H^{\alpha, \beta}$ teatud eeldustel $\alpha, \beta$ ja $\lambda_{1}, \lambda_{2}$ kohta (teoreemid $3.1,3.2,3.3$ ja 3.4 ).

Tartu Riiklik Olikool

Saabus toimetusse

5. V 1961

\section{UBER EINIGE VERALLGEMEINERUNGEN DES SATZES VON MERCER FUR DOPPELFOLGEN}

\section{T. Sõrmus}

\section{Zusammenfassung}

Im Artikel wird der bekannte Satz von Mercer $\left[{ }^{12}\right]$ für den Fall der Doppelfolgen verallgemeinert:

$\left\{\mu_{k}\right\}$ und $\left\{v_{l}\right\}$, sei total-monotone Folgen mit den Eigenschaften $\mu_{0} v_{0}=1$ und $\lim \Delta^{m} \mu_{0}=\lim \Delta^{n} v_{0}=0$; es sei $\lambda>0$ und $h \neq \infty$.Dann folgt aus der Existenz von 


$$
b-\lim \left(\lambda s_{m n}+(1-\lambda) \sum_{k, l=0}^{m-1, n-1}\left({ }_{k}^{m-1}\right)\left({ }^{n-1}\right) \Delta^{m-1-k, n-1-l} \mu_{k} v_{l} s_{k l}\right)=h
$$

auch die Existenz von $b$-lim $s_{m_{n}}$ und die Gleichheit beider Grenzwerte.

$m, n$

Damit ist der Mercersche Satz auch für $H^{\alpha, \beta}$.Verfahren und $C^{\alpha, \beta}$.Verfahren bewiesen.

Ramanujan $\left.{ }^{14}\right]$ hat für den Fall der Doppelfolgen eine andere Verallgemeinerung des Mercerschen Satzes bewiesen:

Es sei $\lambda_{1}>0$ und $\lambda_{2}>0$. Dann folgt aus der Existenz von

$b-\lim _{m, n}\left(\lambda_{1} \lambda_{2} s_{m n}+\frac{\lambda_{1}\left(1-\lambda_{2}\right)}{n+1} \sum_{l=0}^{n} s_{m l}+\frac{\left(1-\lambda_{1}\right) \lambda_{2}}{m+1} \sum_{k=0}^{m} s_{k n}+\frac{\left(1-\lambda_{1}\right)\left(1-\lambda_{2}\right)}{(m+1)(n+1)}, \sum_{k, l=0}^{m, n} s_{k l}\right)=h^{\prime}$

auch die Existenz von $b$ - $\lim s_{m_{n}}$ und die Gleichheit beider Grenzwerte.

$m, n$

Es wird noch gezeigt, dass der Ramanujansche Satz bei speziellen Voraussetzungen über $\alpha, \beta, \lambda_{1}$ und $\lambda_{2}$ für $H^{\alpha, \beta}$-Verfahren und $C^{\alpha, \beta}$-Verfahren noch in Geltung bleibt (die Sätze 3.1, 3.2, 3.3 und 3.4).

Staatsuniversität zu Tartu

Eingegangen am 5. Mai 1961 